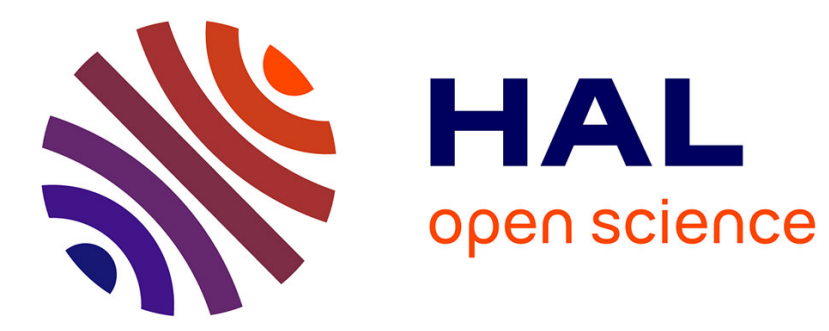

\title{
Idealized models for FEA derived from generative modeling processes based on extrusion primitives
}

Flavien Boussuge, Jean-Claude Léon, Stefanie Hahmann, Lionel Fine

\section{To cite this version:}

Flavien Boussuge, Jean-Claude Léon, Stefanie Hahmann, Lionel Fine. Idealized models for FEA derived from generative modeling processes based on extrusion primitives. 22nd International Meshing Roundtable, Oct 2013, Orlando, United States. hal-00922916v2

\section{HAL Id: hal-00922916 \\ https://hal.inria.fr/hal-00922916v2}

Submitted on 10 Oct 2014

HAL is a multi-disciplinary open access archive for the deposit and dissemination of scientific research documents, whether they are published or not. The documents may come from teaching and research institutions in France or abroad, or from public or private research centers.
L'archive ouverte pluridisciplinaire HAL, est destinée au dépôt et à la diffusion de documents scientifiques de niveau recherche, publiés ou non, émanant des établissements d'enseignement et de recherche français ou étrangers, des laboratoires publics ou privés. 


\title{
Idealized models for FEA derived from generative modeling processes based on extrusion primitives
}

\author{
F. Boussuge ${ }^{1,2}$, J-C. Léon ${ }^{2}$, S. Hahmann ${ }^{2}$, and L. Fine ${ }^{1}$ \\ 1 EADS IW, Suresnes, France \{flavien.boussuge, lionel.fine\}@eads.net \\ 2 Grenoble University - LJK-INRIA, Montbonnot, France \\ jean-claude.leon@grenoble-inp.fr, stefanie.hahmann@inria.fr
}

\begin{abstract}
Shape idealization transformations are very common when adapting a CAD component to FEA requirements. Here, an idealization approach is proposed that is based on generative shape processes used to decompose an initial B-Rep object, i.e. extrusion processes. The corresponding primitives form the basis of candidate sub domains for idealization and their connections conveyed through the generative processes they belong to, bring robustness to set up the appropriate connections between idealized sub domains. Taking advantage of an existing construction tree as available in a CAD software does not help much because it may be complicated to use it for idealization processes. Using generative processes attached to an object that are no longer reduced to a single construction tree but to a graph containing all non trivial construction trees, is more useful for the engineer to evaluate variants of idealization. From this automated decomposition, each primitive is analyzed to define whether it can idealized or not. Subsequently, geometric interfaces between primitives are taken into account to determine more precisely the idealizable sub domains and their contours when primitives are incrementally merged to come back to the initial object.
\end{abstract}

Keywords: B-Rep model, idealization, FEA, additive process, generative shape process

\section{Introduction}

Processing complex objects and determining idealizable areas in a robust manner is still an issue when transforming CAD volumes for FEA and most contributions concentrate on identifying idealizable areas. Producing simple connexions between sub domains is also an issue. Modeling processes can be a good basis to identify idealizable areas but they are difficult to acquire because they are internal to CAD modelers and not available through neutral 
files exchange (STEP, ...). Additionally, they are not unique, i.e. different users may generate different construction trees for the same final shape and even these trees may not be suited to define idealized areas. Using generative processes to decompose an object shape independently of any CAD modeler is a means to obtain a description that is intrinsic to each object [9] while they stand for a set of modeling actions that can be used to identify idealizable sub domains. Processing the geometric interfaces between these sub domains enables the aggregation of sub domains and help updating idealizable sub domains. The review of prior work in these areas is the purpose of the next section.

\section{Prior work}

Different approaches have been proposed to generate automatically idealized models for CAE. Among them, the face-pairing [16, 21] works from nearly parallel faces of CAD models, which produces robust results on a reduced set of configurations, and Medial Axis Transform (MAT) methods work on mesh models, which is more generic, but produce complex geometry in connection areas. More recently, Robinson and Armstrong [17] used the MAT to identify thin regions candidate to idealization. A first step uses a 3D MAT to identify potential volume regions, then the MAT of these regions is analyzed by a second 2D MAT to determine the inner sub-regions which fully meet an aspect ratio between local thickness and MAT dimensions. With this approach, the authors take into account the dimensions associated to the local object thickness. Chong [3] proposes operators to decompose solid models based on concavity shape properties before the mid-surface extraction that reduces the model dimension. However, the solid model decomposition algorithm detects thin configurations if edge pairs exist in the initial model and match an absolute thickness tolerance value. Some volume regions remain not idealized because of the nonexistence of edges-pairs on the initial object.

To reduce the complexity of detection of dimensional reduction areas, Robinson and al. [18] use preliminary CAD information to identify 2D sketches used to generate revolving or sweepable volumes in construction trees. These sketches are analyzed by MAT to determine thin and thick areas. However, in industry, even if the construction tree information exists in a native CAD model, the selected features depend on the designer's modeling choices, which does not ensure to obtain maximal sketches mandatory to get efficient results. Generating construction trees from solid models has been proposed when converting B-rep models into CSG ones [20] using Boolean operations to find one CSG tree but this tree may not produce directly suitable features for idealization. To reduce the complexity of assembly models, Kim et al. [7] propose a multi-resolution decomposition of an initial B-Rep assembly model. These operators simplify the parts by detecting and removing small features and idealize thin volume regions using face pairing. The obtained features are 
structured in a feature tree depending on the level of simplification. This work shows, with three operators, the many possible feature combinations creating multi-resolution models but model abstractions don't meet idealization requirements. Li et al. [10] look for design intents based on recovering symmetries from shape properties. This work is closely related to our method because it iteratively analyses an object but the algorithm produces a unique tree and favors negative features over positive ones. The wrap-around operation proposed by Seo [19] also proposes a multi-step operator but it is restricted to concave features only. Our objective is to favor positive extrusion features to reduce the complexity of the analysis determining idealizable areas.

Our approach is also related to previous work in feature recognition and suppression. Different application domains' requirements lead to a wide variety of feature definitions. In CAE applications, the focus has been set on removing detail features to simplify models before meshing $[4,8]$. A particular domain, mostly studied in the 80-90s is the recognition of machining features. These methods are efficient to recognize and classify negative features as holes, slots or pockets [6]. Han et al. [5] give an overview of the state-of-theart in manufacturing features recognition. Automatic blend features removal, and more precisely finding sequences of blend features in an initial shape, are relevant to FE preprocessing. Regarding blends removal, Zhu and Menq [23] and Venkataraman [22] detect and classify fillet/round features in order to create a suppression order and remove them from a CAD model. In FEM, automatic decomposition of mechanical parts into hex meshable sub-regions create positive feature decompositions. The methods of Lu et al. [13] or Liu and Gadh [12] use edge loops to find convex and sweepable sub-volumes for hex meshing and, more recently, the one proposed by Makem [14] to identify automatically long, slender regions are also close to our work. However, these segmentation algorithms don't aim at producing a construction tree and the features found are extrusions for [14] only. For others, the sub domains may not be extrusions because they should be suited for hex meshing only.

Our work focuses on additive generative processes using extrusion primitives to identify and generate idealized sub domains. Previous methods have shown the possibility of generating modeling processes from an original CAD model. However, the processes generated are unique for a component and often not suited for idealization due to the configurations focusing on particular application areas. In this paper, we propose to generate a construction graph adapted to idealization from extrusion configurations. Sections 3 and 4 describe the main phases of the construction graph generation and section 5 describes how this graph can be used to identify idealizable areas of the initial object. From this first assessment of idealizable areas, a propagation mechanism is described in section 5.3 that follows this 'idealizability' back to the initial model. Then, section 6 illustrates the generation of idealized models with appropriate connections between sub domains. 


\section{Construction graph generation}

\subsection{Modeling context}

As a first step, the focus is placed on a category of mechanical components as modeled using volume modelers that produce B-Rep models. Looking at volume modeling functions in industrial CAD systems, extrusion and revolve operations combined with the addition or removal behavior of a volume domain cover the major range of modeling operations. As a complement, blending radii or chamfers derive from configurations where some of them can be inserted in extrusions or revolutions, i.e. they can be inserted into sketch contours used in extrusion or revolution primitives. But some require specific modeling operations, hence their complementarity (see Figure 1a).
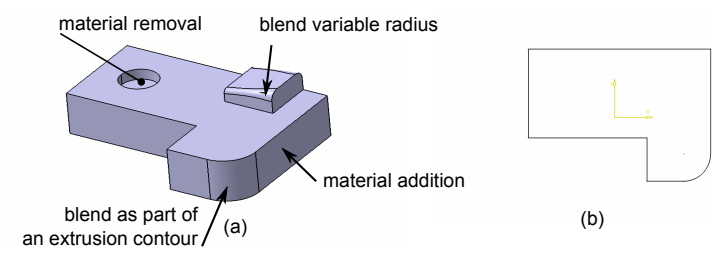

(b)

Fig. 1. a) Set of basic volume modeling operators, b) sketch defining an extrusion primitive in (a).

Still as a first step, we consider the set of modeling functions that incorporate a sketch step in a plane to define at least one closed contour and this contour is reduced to line segments and arcs of circles. These functions cover extrusions and revolutions and this does not restrict significantly the range of mechanical components that can be addressed (see Figure 1b). Combining extrusions and revolutions in a construction tree is equivalent to Boolean operations of type union or subtraction. To start processing engineering components, we focus on extrusion primitives to reduce the complexity of the proposed approach. We assume that the object $M$ analyzed for shape decomposition is free of blending radii and chamfers that cannot be incorporated into sketched contours. Prior work in this field [11] can be used to derive $M$ from the initial object $M_{I}$, possibly with user's interactions.

\subsection{Decomposing an object into sets of extrusion primitives}

Given a target object $M$ to be analyzed, independently of the modeling context stated above, $M$ is obtained through a set of primitives combined together to add or remove material. The B-Rep of $M$ can be seen as the memory of generative processes where primitives are sequentially combined [9]. 
Current CAD modelers are based on strictly sequential processes because the user can hardly generate simultaneous primitives without looking at intermediate results to see how they combine/interact together. Consequently, B-Rep operators in CAD modelers are only binary operators combining the latest primitive generated to the existing shape of $M$ at a stage $t$ of a generative process. Indeed, the decomposition $\mathcal{D}$ of $M$ into extrusion primitives is not bound to a single construction tree but it produces a construction graph $G_{D}$ that contains all possible non trivial construction trees of $M$. To this end, the major concepts and features of $\mathcal{D}$ can be listed as follows. $G_{D}$ is iteratively generated from $M$, 'backward in time', by removing all possible primitives $P_{i}$ until either a single or a set of disconnected extrusion primitive(s) is reached. This termination holds whenever $M$ is effectively decomposable into a set of extrusion primitives. Otherwise, $\mathcal{D}$ is only partial and its termination produces either one or a set of volume partitions describing the most simplest objects $\mathcal{D}$ can reach. Figure 2 summarizes this process. When generating $G_{D}$, we refer to $M=M_{0}$ and evolutions $M_{-j}$ of it backward at the $j^{\text {th }}$ step of $\mathcal{D}$.

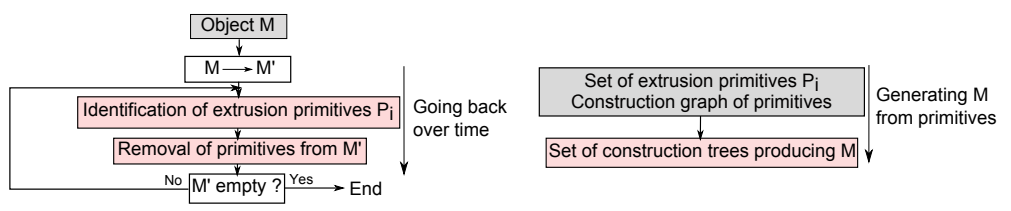

Fig. 2. Overall scheme to obtain construction trees.

$G_{D}$ is an oriented graph where each node contains a set of extrusion primitives $P_{i}$ and arcs are regularized Boolean unions, in our current case. Only such unions are considered presently, not only for simplification purposes but also because these unions are better suited to idealization processes rather than subtractive operators. Indeed, studying the morphology of each $P_{i}$ is sufficient to decide whether a sub domain is idealizable or not. Using regularized unions to propagate idealized $P_{i}$ is the topic addressed in Section 5.3. Incorporating regularized subtractions and unions is left for future work. Figure 3 gives an example of graph obtained on a rather complex object. One can notice that the first steps of the generation of $G_{D}$ of $M$, contain effectively a set of primitives $P_{i}$, each. This is more compact than referring to the combinatorial combinations of dyadic unions as prescribed by industrial CAD modelers. $G_{D}$ is generated automatically with a software application based on Open Cascade software.

Also, this figure highlights the graph structure inherent to the shape of $M$ with the two construction variants taking place between $M_{-4}$ and $M_{-7}$.

Often, the number of possible generative processes producing $M$ can be very large, e.g. even a cube can be obtained from an arbitrary large number of extrusions of arbitrary small extent combined together with a union operator. 


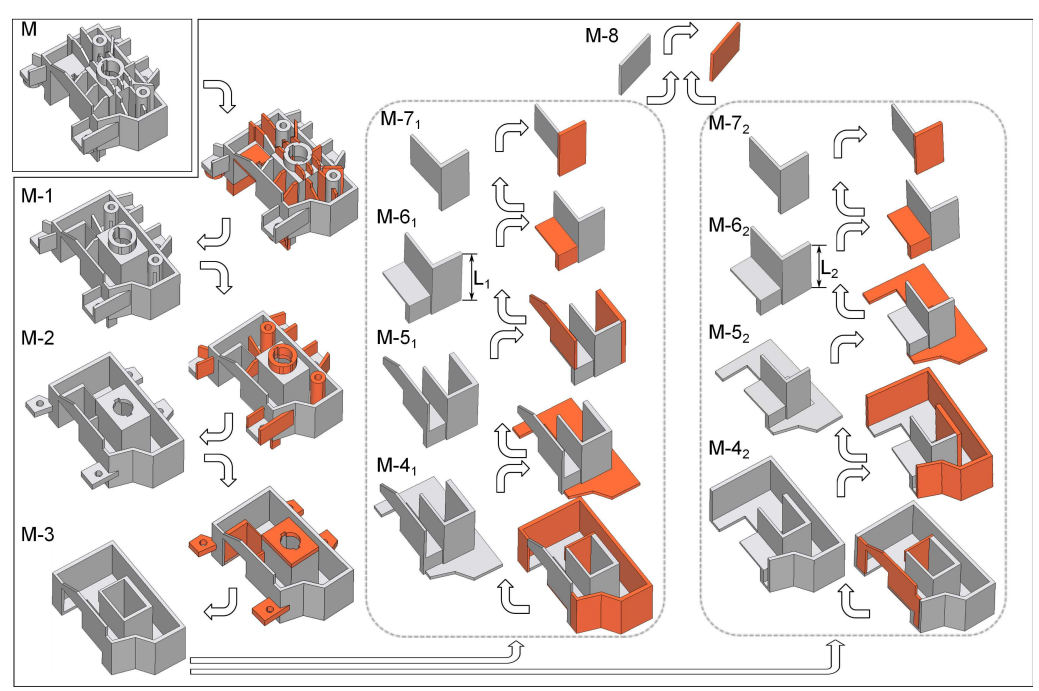

Fig. 3. $G_{D}$ of a component. Orange sub domains indicate the removed primitives $P_{i}$ at each node of $G_{D}$. Label $M_{-j_{k}}$ indicates the step number $j$ when 'going back in time' and the existence of variants $k$, if any. Arrows described the successive steps of $\mathcal{D}$. Arcs of $G_{D}$ are obtained by reversing these arrows to produce construction trees. Steps $M_{-6_{1}}$ and $M_{-6_{2}}$ differ because of distinct lengths $L_{1}$ and $L_{2}$.

Here, we refer to the concept of maximal primitives so that the number of primitives is as small as possible for $M$. As an example, Figure 1a is obtained from three maximal primitives after removing the two blends with variable radii. Maximal primitives mean that the contour of a sketch can be arbitrary complex and extrusion length of each $P_{i}$ is as large as possible.

In order to converge, $\mathcal{D}$ is subjected to two major criteria:

- when a set of primitives $P_{i}$ is removed from $M_{-j}$ to produce $M_{-(j+1)}$, the shape of $M_{-(j+1)}$ must be simpler than that of $M_{-j}$;

- each primitive $P_{i}$ removed from $M_{-j}$ to produce $M_{-(j+1)}$, must be as simple as possible.

Each of these criteria is tightly related to the concept of maximal faces and edges of $\partial M_{-j}$, the boundary of $M_{-j}$. The concept of maximal faces and edges derives from the fact that there is an infinite number of decompositions of $\partial M_{-j}$ that don't change the shape of $M_{-j}$, which is expressed by the Euler's theorem [15]. The concept of maximal faces and edges is mandatory to avoid the side effects of the designer's modeling process, the topological constraints inherent to geometric modelers and some consequences of the parameterization of curves and surfaces describing $\partial M_{-j}$ (see [1] for more details). Generating maximal faces and edges is achieved with a merging operator applied when surfaces adjacent to other are indeed identical, in simple configurations. The outcome of this process is a unique boundary decomposition of $\partial M_{-j}$ 
that is intrinsic to the shape of $M_{-j}$. Therefore, the convergence criteria mentioned previously can rely on the maximal faces and edges of $M_{-j}$ and $P_{i}$ to characterize simple shapes.

Based on the generation principle of $G_{D}$ described previously, the core step of the process is the identification of each $P_{i}$ that can be extracted from $M_{-j}$ to produce $M_{-(j+1)}$. This is the purpose of the next section.

\section{Identifying extrusion primitives in an object}

Starting with the object $M_{-j}$, each $P_{i}$ is identified through two phases:

- $\quad P_{i}$ is visible in $M_{-j}$;

- $\quad P_{i}$ is valid in $M_{-j}$, i.e. the visibility of $P_{i}$ in $M_{-j}$ is invariant w.r.t. the extrusion distance of $P_{i}$ and the geometric interface $I_{G}$ between $P_{i}$ and $M_{-(j+1)}$ is minimal. This refers to the attachment of $P_{i}$ to $M_{-(j+1)}$.

Before addressing the concepts of visibility and attachment, let us first describe the major entities of an extrusion primitive. In an extrusion $P_{i}$ there are two base faces, $F b_{1}$ and $F b_{2}$, that are planar and contain the same sketched contour where the extrusion takes place. Considering extrusions that add volume to a pre-existing object, the edges of $F b_{i}$ are called contour edges and are convex. A convex edge is such that the normals at its adjacent faces define an angle $\alpha$ such that: $0<\alpha<\pi$. When $P_{i}$ belongs to $M_{-j}$, the contour edges along which $P_{i}$ is attached to $M_{-j}$ can be either convex or concave depending on the neighborhood of $P_{i}$ in $M_{-j}$ (see Figure 4a).

In the direction $\mathbf{d}$ of the extrusion, all the edges are straight line segments parallel to each other and orthogonal to $F b_{i}$. These edges are named lateral edges. Faces adjacent to $F b_{i}$ are called lateral faces. They are bounded by four edges, two of them being lateral edges. Lateral edges can be fictive lateral edges when a lateral face coincides with a face of $M_{-j}$ adjacent to $P_{i}$ (see Figure 4a). When lateral faces of $P_{i}$ coincide with adjacent faces in $M_{-j}$, there cannot be edges separating $P_{i}$ from $M_{-(j+1)}$ because of the definition of maximal faces. Such a configuration refers to fictive base edges (see Figure 5 with the definition of primitive $P_{1}$ ).

Visibility. An extrusion primitive $P_{i}$ can be visible in different ways depending on its insertion in a current object $M_{-j}$. The simplest visibility is obtained when $P_{i}$ 's base faces $F b_{i}$ in $M_{-j}$ exist and when at least one lateral edge connects $F b_{i}$ in $M_{-j}$ (see Figures $4 \mathrm{a}$ and 5 (step 1$)$ ).

More generally, the contour of $F b_{1}$ and $F b_{2}$ may differ from each other, see Figure $4 \mathrm{~b}$, or the primitive may have only one base face $F b_{1}$ visible in $M_{-j}$ together with one existing lateral edge that defines the minimal extrusion distance of $F b_{1}$ (see Figure 4c). Our two hypotheses on extrusion visibility thus state as follows. First, at least one base face is visible in $M_{-j}$, i.e. the contour of either $F b_{1}$ or $F b_{2}$ coincides with a subset of the attachment contour of $P_{i}$ in $M_{-j}$. Second, one lateral edge exists that connects $F b_{i}$ in $M_{-j}$. 

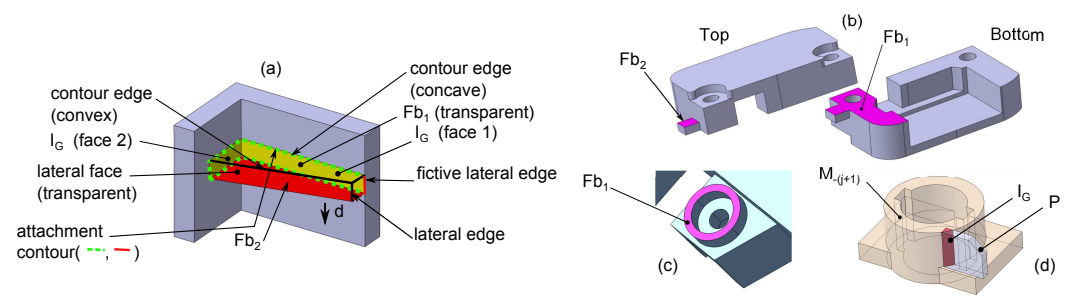

Fig. 4. a) Entities involved in an extrusion primitive. Visible extrusion feature with its two identical base faces $F b_{1}$ and $F b_{2}$. b) Visible extrusion feature with its two different base faces $F b_{1}$ and $F b_{2}$. c) Visible extrusion feature with a unique base face $F b_{1}$. d) Example of geometric interface $I_{G}$ of type volume between $P_{i}$ and $M_{-(j+1)}$.

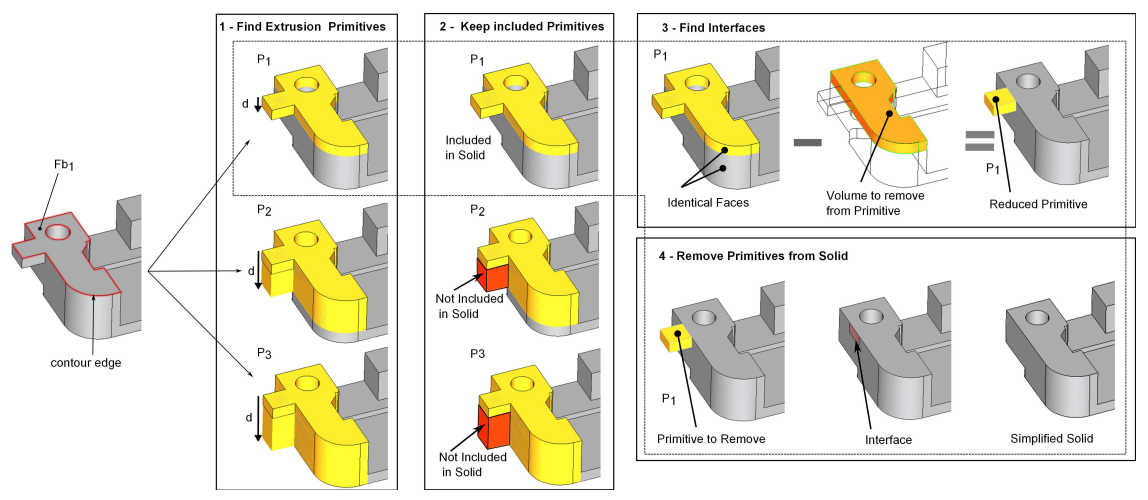

Fig. 5. An example illustrating the major steps for identifying a primitive $P_{i}$ and removing it from the current model $M_{-j}$. Steps 1 and 2 illustrate the influence of the validity of candidate primitives. Step 3 illustrates the effect of the primitive simplicity criterion on $P_{1}$.

Attachment. An extrusion primitive $P_{i}$ is attached to $M_{-j}$ in accordance to its visibility in $M_{-j}$. The attachment defines a geometric interface, $I_{G}$, between $P_{i}$ and $M_{-(j+1)}$, i.e. $I_{G}=P_{i} \cap M_{-(j+1)}$. This interface can be a surface or a volume or both, i.e. a non-manifold model. One of the simplest attachments occurs when $P_{i}$ has its base faces $F b_{1}$ and $F b_{2}$ visible. This means that $P_{i}$ is connected to $M_{-(j+1)}$ through lateral faces only. Consequently, $I_{G}$ is a surface defined by the set of lateral faces not visible in $P_{i}$. Figure 4 a illustrates such a type of interface ( $I_{G}$ contains two faces depicted in yellow).

A simple example of attachment involving a volume interface $I_{G}$ between $P_{i}$ and $M_{-(j+1)}$ is given in Figure 4 d. Notice that the interface between $P_{i}$ and $M_{-(j+1)}$ contains also a surface interface that is not highlighted.

Whatever the category of interface, once $P_{i}$ is identified and its parameters are set (contour and extrusion distance), it is necessary to validate it prior to define its interface. Let $P_{i}$ designates the volume of the reference primitive, 
i.e. the entire extrusion $P_{i}$. To ensure that $P_{i}$ is indeed a primitive of $M_{-j}$, let the necessary condition formally be expressed with regularized Boolean operators between these two volumes (see Figure 5 step 2):

$$
\left(M_{-j} \cup^{*} P_{i}\right)-{ }^{*} M_{-j}=\phi .
$$

This equation states that $P_{i}$ intersects $M_{-j}$ only along the edge loops forming its attachment to $M_{-(j+1)}$, i.e. $P_{i}$ does not cross the boundary of $M_{-j}$ at other location than its attachment. The regularized Boolean subtraction states that limit configurations producing common points, curve segments or surface areas between $P_{i}$ and $M_{-j}$ at any other location than the attachment of $P_{i}$ are acceptable. This condition strongly reduces the number of valid generation processes over time.

The next step is to generate $M_{-(j+1)}$ once $P_{i}$ has been identified and removed from $M_{-j}$. Depending of the type of $I_{G}$, some faces of $P_{i}$ may be added to ensure that $M_{-(j+1)}$ is a volume (see Figure 5 steps 3 and 4 ).

If, in a general setting, there exists several variants of $I_{G}$ to define $M_{-(j+1)}$, these variants always produce a realizable volume, which differs from the halfspace decomposition approaches studied in [20,2] where complements to the halfspaces derived from their initial boundary were needed to produce a realizable volume. Anyhow, all variants of valid $I_{G}$ are processed so that simplest $P_{i}$ and simplest versions of $M_{-(j+1)}$ can be obtained without loosing construction variants of $M$. Other, though less important criteria, can be found in [1] to help classify variants of $M$ that can be of interest for applications differing from idealization.

\section{Performing idealizations from a construction graph}

The purpose of this section is to illustrate how a construction graph $G_{D}$ obtained with the algorithm described at Section 3.2 can be used in shape idealization processes. In fact, idealization processes are high level operations that interact with the concept of detail because the idealization of sub domains triggers their dimensional reduction, which, in turn, influences the shape of areas around $I_{G} \mathrm{~s}$, the geometric interfaces between sub domains. Here, the proposed approach is purely morphological, i.e. it does not depend on discretization parameters like FE sizes. It is divided into two steps. Firstly, each $P_{i}$ of $G_{D}$ is evaluated with respect to an idealization criterion. Secondly, according to $I_{G} \mathrm{~s}$ between $P_{i} \mathrm{~s}$, the 'idealisability' of each $P_{i}$ is propagated in $G_{D}$ through construction trees up to the shape of $M$. As a result, an engineer can evaluate effective idealisable areas. Also, it will be shown how variants of construction trees in $G_{D}$ can influence an idealization process. Because the idealization process of an object is strongly depending on the engineer's know-how, it is the principle of the proposed approach to give the engineer access to the whole range of idealization variants. Finally, some shape details 
will appear subsequently when the engineer will define FE sizes to mesh the idealized representation of $M$.

\subsection{Evaluating sub domains for idealization}

The primitives extracted from the graph are subjected to a morphological analysis to evaluate their adequacy for idealization transformations into plates or shells. Because the primitives are all extrusions and add material, analyzing their morphology can be performed with the MAT [14, 18, 21]. MAT is particularly suited to extrusion primitives having constant thickness since it can be applied in 2D. Further, it can be used to decide whether sub domains can be assigned a plate or shell mechanical behavior. In the present case, the extrusion primitives obtained lead to two distinct configurations (see Figure 6). Figure 6 a shows a configuration with a thin extrusion, i.e. the maximal diameter $\Phi$ obtained with the MAT from $P_{i}$ 's contour is much larger than $P_{i}$ 's thickness defined by the extrusion distance $d$. Then, the idealized sub domain would be a surface parallel to the base face having $P_{i}$ 's contour. Figure $6 \mathrm{~b}$ shows a configuration where the morphology of the sub domain leads to an idealization that would be based on the content of the MAT because $d$ is much larger than $\Phi$.

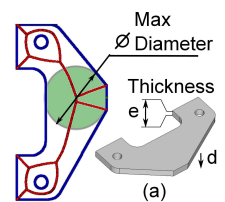

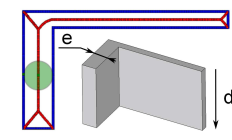

(b)

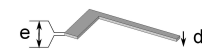

(c)

Fig. 6. Indication of idealization direction of extrusion primitives with 2D MAT applied to their contour.

To idealize a sub domain in mechanics, a commonly accepted reference proportion used to decide whether a sub domain is idealizable or not is a ratio of ten between the in-plane dimensions of the sub domain and its thickness, i.e. $x_{r}=10$. Here, this can be formalized with the morphological analysis of the sub domain obtained from the MAT using: $x=\max ((\max \Phi / d),(d / \max \Phi))$. Consequently, the ratio $x$ is applicable for all morphologies of extrusion sub domains.

Because idealization processes are heavily know-how dependent, using this reference ratio as unique threshold does not seem sufficient to help an engineer analyze sub domains at least because $x_{r}$ does take precisely into account the morphology of the sub domain's contour. To let the engineer tune the morphological analysis and decide when sub domains can/cannot be idealized a second, user-defined threshold, $x_{u}<x_{r}$, is introduced that lies in the interval ] $0, x_{r}[$. Figure $6 \mathrm{~b}$ illustrates a configuration where the morphological analysis 


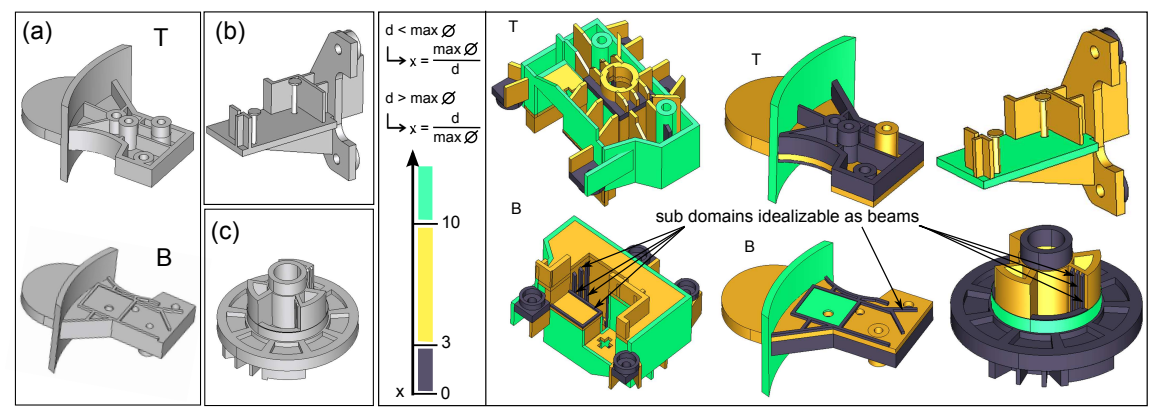

Fig. 7. Idealization analysis of components, the decomposition of one of them is shown at Figure 3. Components a, b, c are new components whose decomposition results reduce to a single tree structure in $G_{D}$. T and B indicate Top and Bottom views of the component, respectively. The decompositions of a and $\mathrm{b}$ are shown in Figure 9. Violet indicates sub domains that cannot be idealized as plates or shells, green ones can be idealized and yellow ones can be subjected to user decision.

does not produce a ratio $x>x_{r}$ though a user might idealize the sub domain as a plate.

Let $x_{u}=3$ be this user-defined value, Figure 7 shows the result of the interactive analysis the user can perform from the graphs $G_{D}$ obtained with the components analyzed in Figures $7 \mathrm{a}, \mathrm{b}, \mathrm{c}$ and 3. Colors interpretation is given in the figure caption. It has to be mentioned that the analysis is applied to $G_{D}$ rather than to a single construction tree structure so that the engineer can evaluate the influence of $\mathcal{D}$ with respect to the idealization processes. However, the result obtained on component of Figure 3 shows that the variants in $G_{D}$ have no influence with respect to the morphological analysis criterion, in the present case. Results on components of Figure 3 and $7 \mathrm{a}, \mathrm{c}$ also show a limit of this criterion because some non-idealizable sub domains (see indications on Figure 7 regarding violet sub domains) are indeed well proportioned to be idealized with beams. Such configurations are clearly calling for complementary criteria that are part of our future work.

These results are already helpful for an engineer but it is up to him or her to evaluate the mechanical effect of $I_{G} \mathrm{~s}$ between primitives $P_{i}$. To help the engineer process the stiffening effects of $I_{G} \mathrm{~s}$, the morphological analysis is extended with a second step as follows.

\subsection{Processing connections between 'idealizable' sub domains}

The morphological analysis of standalone primitives $P_{i}$ is the first application of $G_{D}$. Also, the decomposition obtained can be used to take into account the stiffening effect of interfaces $I_{G}$ between $P_{i}$ when $P_{i}$ are iteratively merged together along their $I_{G}$ up to obtain the whole object $M$. As a result, new sub domains will be derived from $P_{i}$ and the morphological analysis will be 
available on $M$, which will be easier to understand for the engineer. To this end, a taxonomy of connections between extrusion sub domains is mandatory and summarized in Figure 8. This taxonomy refers to parallel and orthogonal configurations for simplicity but these configurations can be extended to process a larger range of angles, i.e. if Figure 8 refers to interfaces of surface type, these configurations can be extended to interfaces of volume type. More specifically, it can be noticed that the configuration where $I_{G}$ is orthogonal to the mid-surfaces of $S_{1}$ and $S_{2}$ both is lacking of robust solutions [16, 21] and other connections can require deviation from mid-surface location to improve the mesh quality. Figure 10b illustrates such configurations and further details will be given in Section 6 .

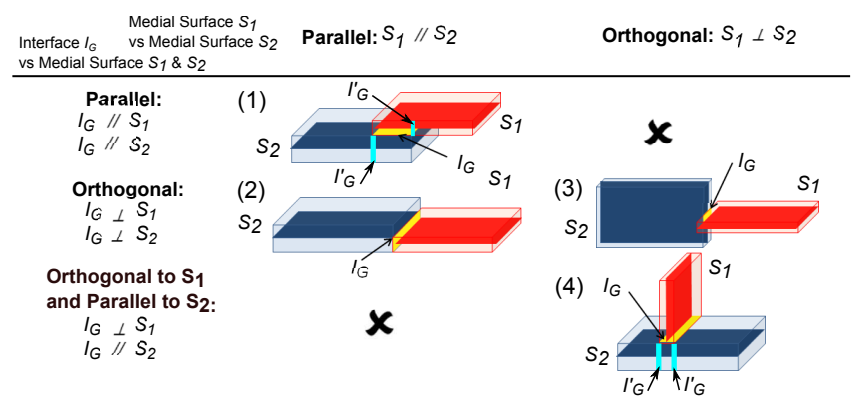

Fig. 8. Taxonomy of connections between extrusion sub domains.

Figure 8 describes all the valid configurations of $I_{G}$ between two sub domains $S_{1}$ and $S_{2}$ when a thickness parameter can be attached to each sub domain, which is presently the case with extrusion primitives. The four valid configurations can be structured into two groups: (1) and (4) form $C_{1}$ and (2) and (3) form $C_{2}$. Configuration (1) of $C_{1}$ is such that the thicknesses $e_{1}$ and $e_{2}$ of $S_{1}$ and $S_{2}$ respectively, are influenced by $I_{G}$, i.e. their overlapping area acts as a thickness increase that stiffens each of them. This stiffening effect can be important to be incorporated into a FE model as a thickness variation to better fit the real behavior of the corresponding structure. Their overlapping area can be assigned to either $S_{1}$ or $S_{2}$ or form an independent sub domain with a thickness $\left(e_{1}+e_{2}\right)$, the sub domains $S_{1}$ and $S_{2}$ get modified as well as their $I_{G}$, producing a configuration of type (2) with a new interface $I_{G}^{\prime}$ that cuts either $S_{1}$ or $S_{2}$ or both depending on the new sub domains created. Similarly, configuration (4) is such that $S_{2}$ can be stiffened by $S_{1}$ depending on the thickness of $S_{1}$ and/or the 2D shape of $I_{G}$ (see examples in Figure 9). In this case, the stiffening effect on $S_{2}$ can partition $S_{2}$ into smaller sub domains and its $I_{G}$ produces a configuration of type (2) with interfaces $I_{G}^{\prime}$ when $S_{2}$ is cut by $S_{1}$. Configuration (1) reduces the areas of $S_{1}$ and $S_{2}$ of constant thicknesses $e_{1}$ and $e_{2}$, which can influence their 'idealizability'. Configuration (4) reduces the area of $S_{2}$ of thickness $e_{2}$ but it is not reducing that of $S_{1}$, which 
influences the 'idealizability' of $S_{2}$ only. As a result, it can be observed that processing configurations in $C_{1}$ produce new configurations that always belong to $C_{2}$. Now, considering configurations in $C_{2}$, none of them is producing stiffening effects as $C_{1}$. Consequently, there is no additional processing needed for $C_{2}$ and processing all configurations in $C_{1}$ produces configurations in $C_{2}$, which outlines the algorithm for processing iteratively interfaces between $P_{i}$.

Figure 8 refers to interfaces $I_{G}$ of surface type. Indeed, $\mathcal{D}$ can produce interfaces of volume type between $P_{i}$. This is equivalent to configurations where $S_{1}$ and $S_{2}$ departs from parallel or orthogonal settings as depicted in Figure 8. Such general configurations can fit into either set $C_{1}$ or $C_{2}$ as follows. In the 2D representations of Figure 8, the outlines of $S_{1}$ and $S_{2}$ define the base faces $F_{b 1}$ and $F_{b 2}$ of each $P_{i}$. What distinguishes $C_{1}$ from $C_{2}$ is the fact that configurations (1) and (4) each contain at least $S_{2}$ such that one of its base face does not intersect $S_{1}$ and this observation applies also for $S_{1}$ in configuration (1). When configurations differ from orthogonal and parallel ones, a first subset of configurations can be classified into one of the four configurations using the distinction observed, i.e. if a base face of either $S_{1}$ or $S_{2}$ does not intersect a base face of its connected sub domain, this configuration belongs to $C_{1}$ and if this property holds for sub domains $S_{1}$ and $S_{2}$ both, the corresponding configuration is of type (1). Some other configurations of type (4) exist but are not detailed here.

\subsection{Extending morphological analyses of sub domains to the whole object}

Now, the purpose is to use the stiffening influence of some connections as analyzed in Section 5.2 to process all the $I_{G}$ between $P_{i}$ to be able to propagate and update the 'idealizability' of each $P_{i}$ when merging $P_{i}$ s. This process ends up with a subdivision of some $P_{i}$ as described in the previous section and a decomposition of $M$ into sub domains, each of them having an evaluation of its 'idealizability' so that the engineer can evaluate more easily the sub domains he or she wants to effectively idealize.

The corresponding algorithm can be synthesized as follows (see algorithm 1). The principle of this algorithm is to classify $I_{G}$ between two $P_{i}$ such that if $I_{G}$ belongs to $C_{1}$ (configurations 1 and 4 in algorithm 1), it must be processed to produce new interface(s) $I_{G}^{\prime}$ and new sub domains that must be evaluated for idealization (procedure Propagate morphology analysis). Depending on the connection configuration between the two primitives $P_{i}$, one of them or both are cut along the contour of $I_{G}$ to produce the new sub domains. Then, the MAT is applied to these new sub domains to update their morphology parameter (procedure MA morphology analysis) that reflects the effect of the corresponding merging operation taking place between the two $P_{i}$ along $I_{G}$ that stiffens some areas of the two primitives $P_{i}$ involved. The algorithm terminates when all configurations of $C_{1}$ have been processed. 


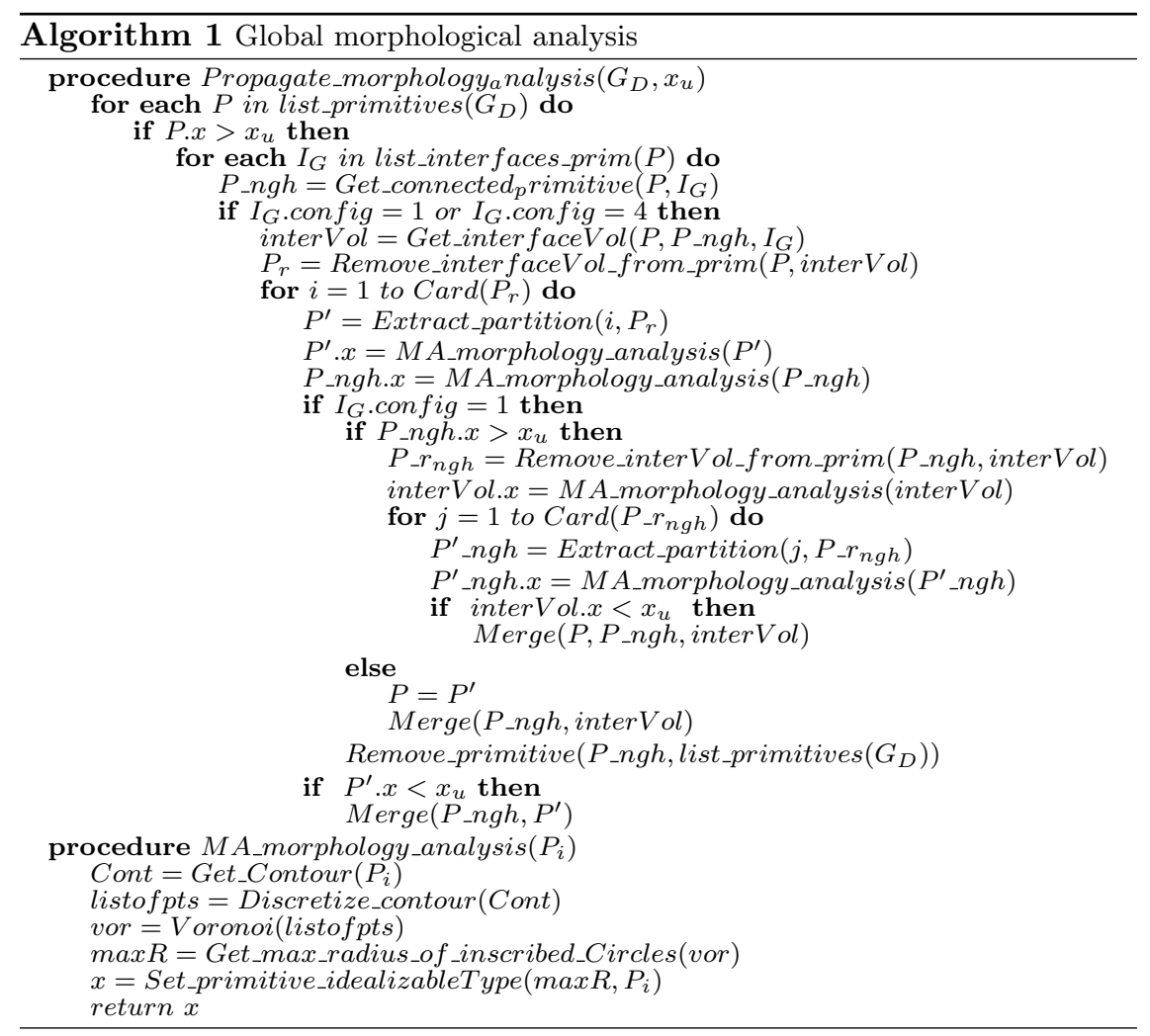

Among the key features of the algorithm, it has to be observed that the influence of the primitive neighbor $P_{n g h}$ of $P_{i}$, is taken into account with the update of $P_{i}$ that becomes $P_{r}$. Indeed, $P_{r}$ can contain several volume partitions, when $\operatorname{Card}\left(P_{r}\right)>1$, depending on the shapes of $P_{i}$ and $P_{n g h}$. Each partition $P^{\prime}$ of $P_{r}$ may exhibit a different morphology than that of $P_{i}$, which is a more precise idealization indication for the engineer. In case of configuration 1, the overlapping area between $P_{n g h}$ and $P_{i}$ must be analyzed too, as well as its influence over $P_{n g h}$ that becomes $P_{r_{n g h}}$. Here again, $P_{r_{n g h}}$ may exhibit several partitions, i.e. $\operatorname{Card}\left(P_{r_{n g h}} \geq 1\right)$, and the morphology of each partition $P_{n g h}^{\prime}$ must be analyzed. If the common volume of $P_{n g h}^{\prime}$ and $P^{\prime}$ is not idealizable, it is merged with either of the stiffest sub domains $P_{n g h}$ or $P_{i}$ to preserve the sub domain the most suited for idealization. In case a partition $P^{\prime}$ of $P_{r}$ is not idealizable in configuration 4, this partition can be merged with $P_{n g h}$ if it has a similar morphological status.

Examples of the extension of the morphological analysis to the whole object $M$ using the interfaces $I_{G}$ between the primitives of $G_{D}$, are given in Figure 9. Figures 9a, b and c depict the construction graphs $G_{D}$ of Figure 7a and b. In the present case, each of these graphs reduce to a single tree struc- 


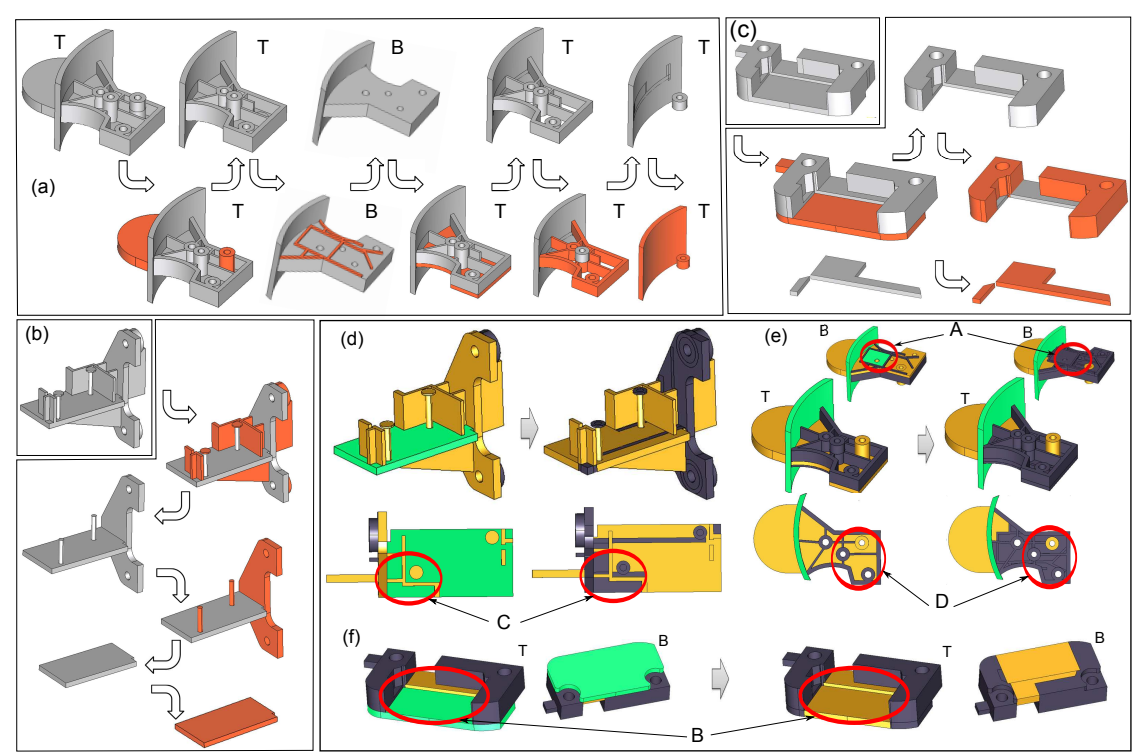

Fig. 9. Propagation of the morphology analysis on $P_{i}$ to the whole object $M$. a, b and c: $G_{D}$ of objects, a and b where depicted in Figure $7 \mathrm{a}$ and b, respectively. d, e and $f$ illustrate the influence of the morphology analysis propagation over the each object b, a, c, respectively when their sub domains are iteratively connected together to form the initial object.

ture. Then, Figures $9 \mathrm{~d}$, e and $\mathrm{f}$ show the sub domain decomposition obtained after processing the interfaces $I_{G}$ between primitives $P_{i}$ of each object $M$. The same figures illustrate also the update of the morphology criterion on each of these sub domains when they are iteratively merged through algorithm 1 to form their initial object $M$. Areas A and B show the stiffening effect of configurations of category (1) on the morphology of sub domains of $M$. Areas C and $\mathrm{D}$ are examples of the subdivision produced with configurations of type (4) and the stiffening effects obtained that are characterized by changes in the morphology criterion values.

After applying algorithm 1, one can notice that every sub domain strictly bounded by one interface $I_{G}$ of $C_{2}$ or by one interface $I_{G}^{\prime}$ produced by this algorithm gives a precise idealization information about an area of $M$. Areas exhibiting connections of type (1) on one or two opposite faces of a sub domain give also precise information, which is the case for examples of Figure 9. However, if there are more piled up configurations of type (1), further analysis is required and will be addressed in the future. 


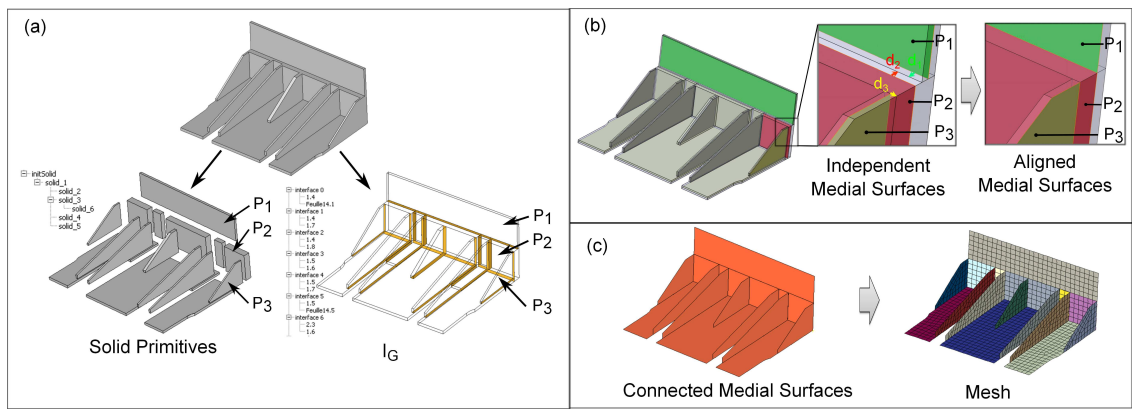

Fig. 10. Idealization process of a component taking advantage of its generative process graph, its corresponding primitives as well as the geometric interfaces between these primitives.

\section{Idealization processes}

Having decomposed $M$ into extrusion primitives $P_{i}$, the location of interfaces $I_{G}$ between $P_{i}$ are precisely identified and can be used to monitor the deviations needed from mid-surfaces to improve the idealization process and take into account the engineer's know-how when preparing a FE model. Particularly, connections with parallel mid-surfaces can be handled with mid-surface repositioning (see $P_{1}$ and $P_{2}$ on Figure 10b) and a corresponding adjustment of the material thickness on both sides of the idealized surface. This is a current practice in linear analysis that has been advantageously implemented using the relative position of extrusions. Similarly, when $S_{1}$ and $S_{2}$ are orthogonal to each other and their $I_{G}$ is located at their boundary (see $P_{2}$ and $P_{3}$ on Figure 10b), either of the mid-surfaces needs to be relocated to avoid meshing narrow areas along one of the sub-domain boundaries (here $P_{3}$ is moved according to $d_{3}$ ). Again, this configuration can be processed using the precise location of $I_{G}$ so that the repositioning operated can stay into $I_{G}$.

Figure 10a illustrates a component with its decomposition through the generative process graph and the corresponding interfaces between its extrusion primitives. This decomposition contains a set of primitive connections of categories discussed in Section 8 and Figure 10b shows the repositioning of mid-surfaces among $P_{1}, P_{2}$ and $P_{3}$ that improves their connections and the overall idealization process. Figure 10c shows the resulting idealized model and its corresponding FE mesh.

\section{Conclusion and future work}

The previous sections have described the main features of a construction graph generation as a backward process to decompose an object into a set of extrusion primitives. This graph is unique for an object and is intrinsic to each object shape because it overcomes modeling, surfaces and topological constraints 
inherent to current CAD modelers. The properties of this graph bring meaningful primitives that can be used as a first step of a morphological analysis. This morphological analysis forms the basis of an analysis of 'idealizability' of primitives. This analysis takes advantage of geometric interfaces between primitives to evaluate stiffening effects that propagate or not across the primitives when they are iteratively merged to regenerate the initial object and locate idealizable sub domains over this object. Though the idealization addressed concentrates on shell and plates, it has been observed that extensions of the morphological analysis can be extended to derive beam idealizations from primitives.

Overall, the construction graph let the engineer access non trivial variants of the shape decomposition into primitives, which can be useful to evaluate variants of idealizations of an object. Then, it has been shown how this decomposition into sub domains and their geometric interfaces can be used to effectively idealize sub domains and take into account some general purpose mesh generation constraints that ensure better quality meshes.

The work described is a first step and needs to be further developed to address a larger range of object shapes as well as more complex construction processes including volume removal operators. Further developments are also required to extend the range of shapes with robust identification of idealizable sub domains and addressing symmetry properties in one next step on that point. These are targets for future work.

\section{Acknowledgments}

This work is carried out in the framework of the ANR project ROMMA (RObust Mechanical Models for Assemblies) referenced ANR-09-COSI-012 and the authors thank the ANR for its financial support. This work is also supported by the ERC Expressive.

\section{References}

1. Boussuge, F., Léon, J.C., Hahmann, S., Fine, L.: Extraction of generative processes from b-rep shapes and application to idealization transformations. CAD (SPM conference) (to appear) (2013)

2. Buchele, S.F., Crawford, R.H.: Three-dimensional halfspace constructive solid geometry tree construction from implicit boundary representations. CAD 36, 1063-1073 (2004)

3. Chong, C.S., Kumar, A.S., Lee, K.H.: Automatic solid decomposition and reduction for non-manifold geometric model generation. CAD 36(13), 1357-1369 (2004)

4. Gao, S., Zhao, W., Lin, H., Yang, F., Chen, X.: Feature suppression based cad mesh model simplification. CAD 42(12), 1178-1188 (2010) 
5. Han, J., Pratt, M., Regli, W.C.: Manufacturing feature recognition from solid models: A status report. IEEE Transactions on Robotics and Automation 16, 782-796 (2000)

6. Joshi, S., Chang, T.C.: Graph-based heuristics for recognition of machined features from a 3d solid model. CAD 20(2), 58-66 (1988)

7. Kim, S., Lee, K., Hong, T., Kim, M., Jung, M., Song, Y.: An integrated approach to realize multi-resolution of b-rep model. In: Proceedings of the 2005 ACM symposium on Solid and physical modeling, SPM '05, pp. 153-162 (2005)

8. Lee, K.Y., Armstrong, C.G., Price, M.A., Lamont, J.H.: A small feature suppression/unsuppression system for preparing b-rep models for analysis. In: Proceedings of the 2005 ACM symposium on Solid and physical modeling, SPM '05, pp. 113-124 (2005)

9. Leyton, M.: A Generative Theory of Shape. Lecture Notes in Computer Science LNCS 2145. Springer-Verlag (2001)

10. Li, M., Langbein, F.C., Martin, R.R.: Constructing regularity feature trees for solid models. In: Proc. Geometric Modeling and Processing; LNCS, pp. 267-286 (2006)

11. Lim, T., Medellin, H., Torres-Sanchez, C., Corney, J.R., Ritchie, J.M., Davies, J.B.C.: Edge-based identification of dp-features on free-form solids. IEEE Trans. PAMI 27(6) (2005)

12. Liu, S.S., Gadh, R.: Automatic hexahedral mesh generation by recursive convex and swept volume decomposition. In: 6th International Meshing Roundtable, Sandia National Laboratories, pp. 217-231 (1997)

13. Lu, Y., Gadh, R., Tautges, T.J.: Feature based hex meshing methodology: feature recognition and volume decomposition. CAD 33(3), 221-232 (2001)

14. Makem, J.E., Armstrong, C.G., Robinson, T.T.: Automatic decomposition and efficient semi-structured meshing of complex solids. In: Proceedings of the 20th International Meshing Roundtable, pp. 199-215 (2012)

15. Mäntylä, M.: An introduction to solid modeling. Computer Science Press, College Park, MD (1988)

16. Rezayat, M.: Midsurface abstraction from 3d solid models: general theory and applications. CAD 28(1), 905-915 (1996)

17. Robinson, T.T., Armstrong, C., Fairey, R.: Automated mixed dimensional modelling from 2d and 3d cad models. Finite Elem. Anal. Des. 47(2), 151-165 (2011)

18. Robinson, T.T., Armstrong, C.G., McSparron, G., Quenardel, A., Ou, H., McKeag, R.M.: Automated mixed dimensional modelling for the finite element analysis of swept and revolved cad features. In: Proceedings of the $2006 \mathrm{ACM}$ symposium on Solid and physical modeling, SPM '06, pp. 117-128 (2006)

19. Seo, J., Song, Y., Kim, S., Lee, K., Choi, Y., Chae, S.: Wrap-around operation for multi-resolution cad model. CAD and Applications 2(1-4), 67-76 (2005)

20. Shapiro, V., Vossler, D.L.: Separation for boundary to csg conversion. ACM Trans. Graph. 12(1), 35-55 (1993)

21. Sheen, D.P., Son, T.G., Myung, D.K., Ryu, C., Lee, S.H., Lee, K., Yeo, T.J.: Transformation of a thin-walled solid model into a surface model via solid deflation. CAD 42(8), 720-730 (2010)

22. Venkataraman, S., Sohoni, M., Rajadhyaksha, R.: Removal of blends from boundary representation models. In: Proceedings of the seventh ACM symposium on Solid modeling and applications, SMA '02, pp. 83-94 (2002)

23. Zhu, H., Menq, C.H.: B-rep model simplification by automatic fillet/round suppressing for efficient automatic feature recognition. CAD 34(2), 109 - 123 (2002) 
